\title{
Generalized Ghost Dark Energy Model with Varying $G$
}

\author{
Leila Yousefi ${ }^{1}$, Ahmad Sheykhi $^{2, *}$ \\ ${ }^{1}$ Department of Sciences, Fasa Branch, Islamic Azad University, Fasa, Iran \\ ${ }^{2}$ Physics Department and Biruni Observatory, Shiraz University, Shiraz 71454, Iran \\ *Corresponding Author: asheykhi@shirazu.ac.ir
}

Copyright (C)2013 Horizon Research Publishing All rights reserved.

\begin{abstract}
The cosmological implications of the generalized ghost dark energy model in the presence of varying gravitational constant is investigated. In particular, evolution of the cosmological parameters such as the equation of state and the deceleration parameters are studied. The investigation is also generalized to the more general case where there is a bulk viscosity in the cosmic fluids. It is shown that by taking $0<\triangle_{G} \leq 0.07, \xi=0.01$ and assuming $\Omega_{D}^{0}=0.73$ for the present time, we find $-0.80<w_{D}^{0} \leq-0.78$ and $-0.41<q_{0} \leq-0.36$ for the present ranges of the equation of state and the deceleration parameters. This values are consistent with the recent observations.
\end{abstract}

Keywords Generalized, Dark Energy, Ghost, Varying, Gravitational, Constant

\section{Introduction}

Nowadays there is an ample consensus on the observational side that our Universe is experiencing a phase of accelerated expansion likely driven by some unknown energy component usually dubbed "dark energy" [1]. Recent astronomical observations indicate that nearly 70 percent of the Universe consists of dark energy with negative pressure [1]. Disclosing the nature and the origin of such a dark energy has been one of the biggest challenges of the modern cosmology.

Many theoretical candidates have been proposed as dark energy, among them, those which originate from fundamental theory such as quantum gravity, has arisen a lot of enthusiasm, recently. Two examples of such models are holographic and agegraphic dark energy models $[2,3,4,5,6,7,8]$. For a comprehensive review on dark energy models see [9]. In order to explain the acceleration of the cosmic expansion, in most of these dark energy models, one needs to consider a new degree of freedom or a new parameter.

Among various models of dark energy, the so called ghost dark energy (GDE) has attracted a lot of interests in recent years. The origin of dark energy, in this model, comes from Veneziano ghosts in QCD theory [10, 11, 12, 13]. Indeed, the contribution of the ghosts field to the vacuum energy in curved space or time-dependent background can be regarded as a possible candidate for the dark energy [14, 15]. The magnitude of this vacuum energy is of order $\Lambda_{Q C D}^{3} H$, where $H$ is the Hubble parameter and $\Lambda_{Q C D}^{3}$ is $Q C D$ mass scale. With $\Lambda_{\mathrm{QCD}} \sim 100 M e V$ and $H \sim 10^{-33} \mathrm{eV}, \Lambda_{\mathrm{QCD}}^{3} H$ gives the right order of magnitude $\sim\left(3 \times 10^{-3} \mathrm{eV}\right)^{4}$ for the observed dark energy density [14]. The advantages of GDE model compared to other dark energy models is that it is totally embedded in standard model and general relativity, one needs not to introduce any new parameter, new degree of freedom or to modify gravity. The dynamical behavior of GDE model in flat universe has been explored in [16]. The studies were also generalized to the Universe with spacial curvature [17]. The instability of GDE against perturbations was investigated in [18]. In [19, 20] the correspondence between GDE and scalar field models of dark energy were established. Other features of the GDE model have been investigated in Refs. [21, 22, 23, 24, 25, 26]. However, it was argued that the vacuum energy of the Veneziano ghost field in QCD is of the form $H+O\left(H^{2}\right)[27]$, where the sub-leading term $\mathrm{H}^{2}$ should be considered especially at the early stage of the Universe acting as the early dark energy [29]. It was also shown that considering this term leads to a better agreement with observational data [29]. The GDE with sub-leading term $H^{2}$ is usually called generalized ghost dark energy (GGDE) and various aspects of it has been investigated in the literature [30, 31].

It seems likely that one can only talk about variations of dimensionless quantities, such as the fine structure constant. In other words, the fundamental constants may not be constant at all. Indeed, there have been many 
attempts in the literature to theoretically justified a varying gravitational constant. For example, in Brans-Dicke theory the gravitational constant is replaced by a scalar field coupling to gravity through a new parameter, and it has been generalized to various forms of scalar-tensor theories [32], leading to a considerably broader range of variable- $G$ theories. In addition, justification of a varying Newton's constant has been established with the use of conformal invariance and its induced local transformations [33]. Finally, a varying $G$ can arise perturbatively through a semiclassical treatment of Hilbert-Einstein action [34], non-perturbatively through quantum gravitational approaches within the "Hilbert-Einstein truncation" [35], or through gravitational holography [36, 37]. Observations of Hulse-Taylor binary pulsar B1913 + 16 lead to the estimation $\dot{G} / G \sim 2 \pm 4 \times 10^{-12} \mathrm{yr}^{-1}$ [38, 39], while helio-seismological data provide the bound $-1.6 \times 10^{-12} \mathrm{yr}^{-1}<\dot{G} / G<0$ [40]. Similarly, Type Ia supernova observations give the best upper bound of the variation of $G$ as $-10^{-11} \mathrm{yr}^{-1} \leq \frac{\dot{G}}{G}<0$ at redshifts $z \simeq 0.5$ [41], while astereoseismological data from the pulsating white dwarf star G117-B15A lead to $\left|\frac{\dot{G}}{G}\right| \leq 4.10 \times 10^{-11} y r^{-1}$ [42]. See also [43] for various bounds on $\dot{G} / G$ from observational data, noting that all these measurements are valid at relatively low redshifts, i.e $z<3.5$. Since the limits in $G$-variation are given for $\dot{G} / G$ in units $y r^{-1}$, and since $\dot{G} / G=H G^{\prime} / G$, we can estimate $\Delta_{G}=G^{\prime} / G$ substituting the value of $H$ in $y r^{-1}[44,45]$.

Previously, some dark energy models with varying gravitational constant have been explored in the literature. For example, the holographic dark energy model in the presence of varying $G$ were studied in [44, 45], while the new agegraphic dark energy [46] and GDE model with varying gravitational constant were investigated in [47]. However, till now, in all investigations on GGDE model a constant $G$ have been considered. In this paper, we would like to study the effects of the time varying gravitational constant in the Universe filled with GGDE and dark matter.

This paper is organized as follows. In section II, we study the GGDE model with varying gravitational constant. In section III, we extend our study to the case where there is a viscosity in the bulk fluid. The last section is devoted to concluding remarks.

\section{GGDE model with varying gravitational constant}

Consider a flat Friedmann-Robertson-Walker (FRW) universe. The first Friedmann equation is

$$
H^{2}=\frac{8 \pi G}{3}\left(\rho_{m}+\rho_{D}\right)
$$

where $H=\dot{a} / a$ is the Hubble parameter, $\rho_{m}$ and $\rho_{D}$ are, respectively, the energy densities of pressureless matter and dark energy, which satisfy the conservation equations

$$
\begin{aligned}
\dot{\rho}_{m}+3 H \rho_{m} & =0 \\
\dot{\rho}_{D}+3 H \rho_{D}\left(1+w_{D}\right) & =0 .
\end{aligned}
$$

where $w_{D}=p_{D} / \rho_{D}$ is the equation of state parameter of dark energy. The energy density of GGDE is written in the form [29]

$$
\rho_{D}=\alpha H+\beta H^{2},
$$

where $\alpha$ and $\beta$ are two constant. The constant $\alpha$ is of order $\Lambda_{\mathrm{QCD}}^{3}$ where $\Lambda_{\mathrm{QCD}}$ is QCD mass scale. In the GGDE, $\beta$ is a free parameter and can be adjusted for better agreement with observations. Defining the fractional energy density parameters as

$$
\Omega_{m}=\frac{8 \pi G \rho_{m}}{3 H^{2}}, \quad \Omega_{D}=\frac{8 \pi G \rho_{D}}{3 H^{2}},
$$

the Friedmann equation (1) can be rewritten as

$$
\Omega_{m}+\Omega_{D}=1
$$

If we take the derivative of (4) with respect to the cosmic time, we arrive at

$$
\dot{\rho}_{D}=\dot{H}(\alpha+2 \beta H) \text {. }
$$

Next, we take the time derivative of Friedmann equation (1), after using the continuity equations (2) and (3), as well as relations (5) and $\dot{G}=H G^{\prime}$, we get

$$
\frac{\dot{H}}{H^{2}}=\frac{\triangle_{G}}{2}-\frac{3}{2}\left(1+\Omega_{D} w_{D}\right),
$$

where $\triangle_{G}=G^{\prime} / G$ arises from the varying nature of $G$ and the prime denotes derivative with respect to $x=\ln a$. Combining relations (7) and (8) with continuity equation (3), and solving the resulting equation for $w_{D}$, we find

$$
w_{D}=\frac{\xi-\Omega_{D}-\triangle_{G}\left(\xi+\Omega_{D}\right) / 3}{\Omega_{D}\left(2-\Omega_{D}-\xi\right)},
$$




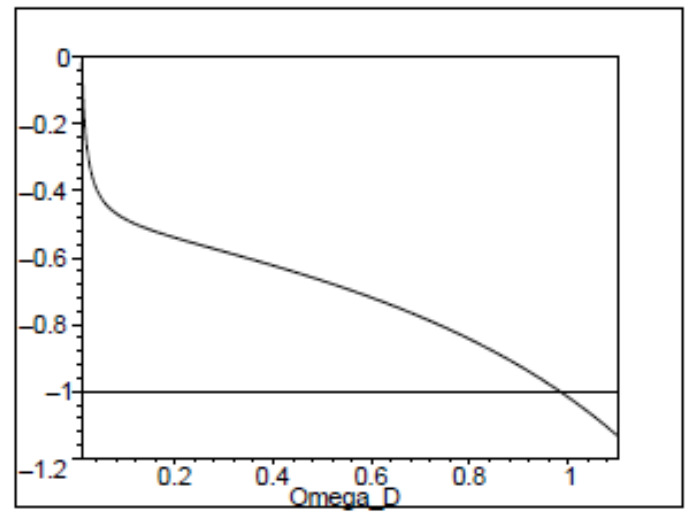

Figure 1. The behaviour of $w_{D}$ versus $\Omega_{D}$ for $\xi=0.01$ and $\triangle_{G}=0.05$

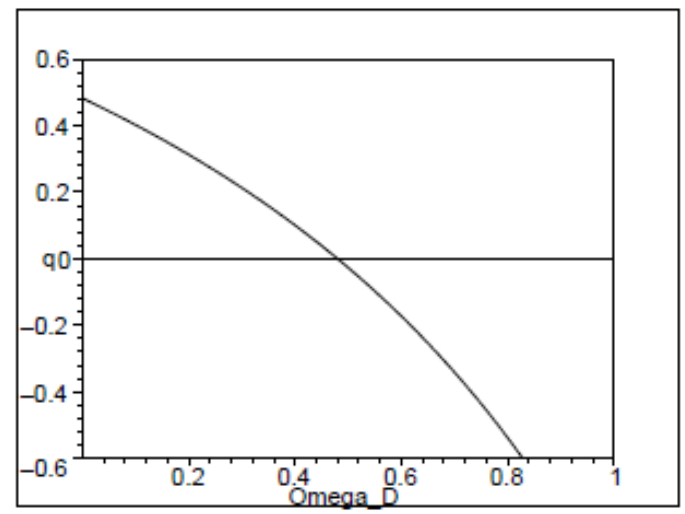

Figure 2. The behaviour of $q$ versus $\Omega_{D}$ for $\xi=0.01$ and $\triangle_{G}=0.05$

where $\xi=8 \pi G \beta / 3$. When $\triangle_{G}=0$, we obtain the equation of state parameter of GGDE [31],

$$
w_{D}=\frac{\xi-\Omega_{D}}{\Omega_{D}\left(2-\Omega_{D}-\xi\right)} .
$$

In the limiting case $\xi=0$, one finds the equation of state parameter of original GDE with varying gravitational constant in a flat universe [47]. Another quantity which is important in studying the evolution of the universe is the deceleration parameter. When the deceleration parameter is combined with the Hubble parameter and the dimensionless density parameters form a set of useful parameters for the description of the astrophysical observations. The deceleration parameter is defined as

$$
q=-1-\frac{\dot{H}}{H^{2}} .
$$

Substituting relation (8) into (11), we find

$$
q=\frac{1}{2}-\frac{\triangle_{G}}{2}+\frac{3}{2} \Omega_{D} w_{D}
$$

Using Eq. (9), we obtain

$$
q=\frac{1}{2}-\frac{3}{2}\left(\frac{\Omega_{D}-\xi}{2-\Omega_{D}-\xi}\right)-\frac{\triangle_{G}}{2-\Omega_{D}-\xi} .
$$

If we take $0<\triangle_{G} \leq 0.07$ [43] and assuming $\Omega_{D}=0.73$ for the present time and $\xi=0.01$, we get $-0.80<$ $w_{D} \leq-0.78$ and $-0.41<q \leq-0.36$ which are consistent with observations $[48,49,50]$. The behaviour of $w_{D}$ and $q$ versus the dimensionless density parameter $\Omega_{D}$ are shown in figures 1 and 2 . These figures show that the transition from deceleration to the acceleration phase take places around $\Omega_{D} \simeq 0.48$. On the other hand, in the late time where the GGDE dominates and for $0.983 \leq \Omega_{D} \leq 1$, the equation of state parameter, $w_{D}$, can cross the phantom line, i. e., $w_{D}<-1$. Finally, we obtain the equation of motion of GGDE with varying gravitational constant. For this purpose, we first take the time derivative of relation

$$
\Omega_{D}=\frac{8 \pi G \rho_{D}}{3 H^{2}}=\frac{8 \pi G}{3 H}(\alpha+\beta H) .
$$

After using relation $\dot{\Omega}_{D}=H \Omega_{D}^{\prime}$, as well as relation (12), we reach

$$
\frac{d \Omega_{D}}{d \ln a}=\triangle_{G} \Omega_{D}+\left(\Omega_{D}-\xi\right)(1+q) .
$$


Substituting $q$ from Eq. (13), we arrive at

$$
\frac{d \Omega_{D}}{d \ln a}=\triangle_{G} \Omega_{D}+3\left(\Omega_{D}-\xi\right)\left[\frac{1-\Omega_{D}-\triangle_{G} / 3}{2-\Omega_{D}-\xi}\right] .
$$

\section{Varying gravitational constant and viscous GGDE}

Next we generalize the investigation to the viscous GGDE model with varying gravitational constant. The cosmological model with viscous cosmic fluid was first studied in [51]. A special branch of viscous cosmology is to investigate how the bulk viscosity can influence the future singularity, commonly called the Big Rip, when the fluid is in the phantom state corresponding to $w_{D}<-1$. A lot of works have been done in this direction [52, 53, 54]. In particular, it was first pointed out in [52] that the presence of a bulk viscosity proportional to the Hubble expansion $H$ can cause the fluid to pass from the quintessence region into the phantom region and thereby inevitably lead to a future singularity. Dark energy with bulk viscosity has a peculiar property to cause accelerated expansion of phantom type in the late evolution of the universe $[52,53]$. The bulk viscosities are negligible for non-relativistic and ultra-relativistic fluids but are important for the intermediate cases. In viscous cosmology, shear viscosities arise in relation to space anisotropy while the bulk viscosity accounts for the space isotropy [52]. In the presence of viscosity the energy-momentum tensor of the viscous fluid is given by

$$
T_{\mu \nu}=\rho_{D} u_{\mu} u_{\nu}+\tilde{p}_{D}\left(g_{\mu \nu}+u_{\mu} u_{\nu}\right)
$$

where

$$
\tilde{p}_{D}=p_{D}-3 H \zeta
$$

is the effective pressure of dark energy and $u_{\mu}$ is the four-velocity vector. Here $\zeta$ is the bulk viscosity coefficient which is chosen such that $\zeta>0$ in order to get positive entropy production in conformity with second law of thermodynamics [55]. The conservation equation for the viscous GGDE reads

$$
\dot{\rho}_{D}+3 H\left(\rho_{D}+\tilde{p}_{D}\right)=0
$$

where it can also be written as

$$
\dot{\rho}_{D}+3 H \rho_{D}\left(1+w_{D}\right)=9 H^{2} \zeta
$$

Inserting Eqs. (7) and (8) into (20), we find

$$
w_{D}=-\frac{1}{\Omega_{D}\left(2-\Omega_{D}-\xi\right)}\left[\Omega_{D}-\xi+\frac{\triangle_{G}}{3}\left(\Omega_{D}+\xi\right)-\frac{6 \zeta}{\alpha}\left(\Omega_{D}-\xi\right)\right] .
$$

In the limiting case $\zeta=0$, one recovers the result of the previous section. In the presence of viscosity the deceleration parameter and evolutionary behaviour of GGDE are still given by Eqs. (12) and (15), where $w_{D}$ is now obtained from (21). It is interesting to study the pure effect of viscosity on GGDE model in flat universe. In this case we set $\triangle_{G}=0$ and the equation of state parameter reduces to

$$
w_{D}=-\frac{\Omega_{D}-\xi}{\Omega_{D}\left(2-\Omega_{D}-\xi\right)}\left(1-\frac{6 \zeta}{\alpha}\right) .
$$

For the late time $\xi<\Omega_{D}$ and $\Omega_{D}<2-\xi$, so for $\zeta<\alpha / 6$ we have $w_{D}<0$. At the present time, if we take $\Omega_{D}^{0}=0.73$ and $\xi=0.01$, the accelerated expansion can be achieved, namely $w_{D}<-1 / 3$, provided $\zeta<0.096 \alpha$. This implies that in an accelerating universe, the bulk viscosity coefficient $\zeta$ is much smaller than the ghost energy density coefficient.

\section{Summary and discussion}

It is a general belief that one can only talk about variations of dimensionless quantities, such as the fine structure constant $\alpha_{e}=e^{2} /\left(4 \pi \varepsilon_{0} \hbar c\right)$. This is due to the fact that physical units are quite arbitrary, and each individual constant can be removed through a suitable choice of units. The simplest example is the speed of light, $c$, whose variation is now manifestly unmeasurable because of its designation as a fixed constant relating the definition of time and distance units. Dimensionless quantities, on the other hand, are independent of the choice of units and so should admit the possibility of genuinely observable changes. There are significant indications that Newton's gravitational constant, $G$, can vary, being a function of time or equivalently of the scale factor [56]. Indeed, there have also been many studies aimed at constraining variations of Newton's gravitational constant, $G$. The role of $G$-variation will be expressed through the pure number $\Delta_{G} \equiv G^{\prime} / G$, which will be extracted from observations [45].

On the other hand, the dark energy puzzle has been one of the biggest challenges of the modern cosmology in the past decade. However, in most studies on dark energy models, people usually assume the gravitational constant 
$G$ does not vary with time. However, there are some evidences which indicate that a varying gravitational constant can give a better fit with observations [45]. Therefore, it is quite interesting to study the dark energy models in the presence of varying gravitational constant. This investigation can reveal the effect of varying $G$ on the evolution of the universe and in particular its effect on the acceleration of the Universe expansion. In this paper, we investigated the GGDE model in the presence of varying gravitational constant. We obtained the equation of state and the deceleration parameters of the model. Taking $0<\triangle_{G} \leq 0.07, \xi=0.01$, and assuming $\Omega_{D}^{0}=0.73$ for the present time, we found $-0.80<w_{D}^{0} \leq-0.78$ and $-0.41<q_{0} \leq-0.36$ for the present ranges of the equation of state and the deceleration parameters. This values are consistent with the recent observations [48, 49, 50]. We also extended the investigations to the case where there is a bulk viscosity in the presence of GGDE. For pure viscous ghost energy, the present acceleration of the Universe expansion can be derived provided we take $\zeta<0.096 \alpha$, where $\zeta$ and $\alpha$ are, respectively, the bulk viscosity coefficient and the ghost energy density coefficient.

Finally, we would like to mention that the viscous GGDE model with time varying gravitational constant, has four free parameters, namely the GDE parameters $\alpha$ and $\xi$, the viscosity parameter $\zeta$, and the $G$-varying parameter $\Delta_{G}$. One way to reduce the parameters of the model is to fix some parameters and only allow other parameters to constraint with observational data. In this work, we only restricted our study to limited observational data. Having the wide range of cosmological data at hand, we will further constrain our model parameter space in the future and test the viability of this model.

\section{Acknowledgements}

This work has been supported financially by Islamic Azad University, Fasa Branch, Fasa, Iran.

\section{REFERENCES}

[1] A.G. Riess, et al., Astron. J. 116 (1998) 1009;

S. Perlmutter, et al., Astrophys. J. 517 (1999) 565;

S. Perlmutter, et al., Astrophys. J. 598 (2003) 102;

A.G. Riess, et al., Astrophys. J. 607, (2004) 665.

[2] A. Cohen, D. Kaplan, A. Nelson, Phys. Rev. Lett. 82 (1999) 4971;

M. Li, Phys. Lett. B 603 (2004) 1;

Q. G. Huang, M. Li, JCAP 0408 (2004) 013;

M. Li, Phys. Lett. B 603, 1 (2004);

B. Wang, E. Abdalla, R. K. Su, Phys. Lett. B 611 (2005) 21;

J. Y. Shen, B. Wang, E. Abdalla, R. K. Su, Phys. Lett. B 609 (2005) 200.

[3] E. J. Copeland, M. Sami and S. Tsujikawa, Int. J. Mod. Phys. D 15 (2006) 1753.

[4] A. Sheykhi, B. Wang and N. Riazi, Phys. Rev. D 75 (2007) 123513.

[5] A. Sheykhi, Phys. Lett. B 680 (2009) 113.

[6] A. Sheykhi, Phys. Lett. B 682 (2010) 329;

A. Sheykhi, Int. J. Mod. Phys. D 18, No. 13 (2009) 2023;

A. Sheykhi, Phys. Rev. D 81 (2010) 023525;

A. Sheykhi, Int. J. Mod. Phys. D Vol. 19, No. 3 (2010) 305;

A. Sheykhi, Mubasher Jamil, Phys. Lett. B 694 (2011) 284

[7] A. Sheykhi, Phys. Scr. 84 (2011) 065904;

E. Ebrahimi and A. Sheykhi, Phys. Scr. 84 (2011) 045016;

A. Sheykhi and M. Jamil, Gen. Relativ. Gravit., 43 (2011) 2661;

K. Karami, et. al., Gen. Rel. Grav. 43 (2011) 27;

M. Jamil and A. Sheykhi, Int. J. Theor. Phys. 50 (2011) 625.

[8] R. G. Cai, Phys. Lett. B 657 (2007) 228;

H. Wei and R. G. Cai, Phys. Lett. B 660 (2008) 113.

[9] M. Li, X. D. Li, S. Wang, Y. Wang, Commun. Theor. Phys. 56 (2011) 525.

[10] K. Kawarabayashi and N. Ohta, Nucl. Phys. B 175 (1980) 477.

[11] E. Witten, Nucl. Phys. B 156 (1979) 269;

G. Veneziano, Nucl. Phys. B 159 (1979) 213.

[12] C. Rosenzweig, J. Schechter and C. G. Trahern, Phys. Rev. D 21 (1980) 3388.

[13] P. Nath and R. L. Arnowitt, Phys. Rev. D 23 (1981) 473. 
[14] N. Ohta, Phys. Lett. B 695 (2011) 41.

[15] F. R. Urban and A. R. Zhitnitsky, Phys. Lett. B 688 (2010) 9;

F. R. Urban and A. R. Zhitnitsky, Phys. Rev. D 80 (2009) 063001;

F. R. Urban and A. R. Zhitnitsky, JCAP 0909 (2009) 018.

[16] R.G. Cai, Z.L. Tuo, H.B. Zhang, arXiv:1011.3212.

[17] A. Sheykhi, M.Sadegh Movahed, Gen Relativ Gravit 44 (2012) 449.

[18] E. Ebrahimi and A. Sheykhi, Int. J. Mod. Phys. Vol. 20, No. 12 (2011) 2369.

[19] A. Sheykhi, M. Sadegh Movahed, E. Ebrahimi, Astrophys Space Sci 339 (2012) 93.

[20] A. Sheykhi, A. Bagheri, Euro. Phys. Lett. 95 (2011) 39001.

[21] E. Ebrahimi and A. Sheykhi, Phys. Lett. B 706 (2011) 19.

[22] A. Rozas-Fernandez, Phys. Lett. B 709 (2012) 313.

[23] K. Karami, M. Mousivand, Int. J. Mod. Phys. D 22 (2013) 1350018.

[24] A. Khodam-Mohammadi, M. Malekjani, M. Monshizadeh, Mod. Phys. Lett. A 27 (2012) 1250100.

[25] M. Malekjani, A. Khodam-Mohammadi, Astrophys. Space Sci. 343 (2013) 451461.

[26] Chao-Jun Feng, Xin-Zhou Li, Xian-Yong Shen, Phys. Rev. D 87 (2013) 023006.

[27] A. R. Zhitnitsky, Phys. Rev. D 86 (2012) 045026.

[28] M. Maggiore, L. Hollenstein, M. Jaccard and E. Mitsou, Phys. Lett. B 704 (2011) 102.

[29] R. G. Cai, Z. L. Tuo, Y. B. Wu, Y. Y. Zhao, Phys.Rev. D 86 (2012) 023511.

[30] A. Sheykhi, E. Ebrahimi and Y. Yousefi, Can. J. Phys. 91 (2013) 662.

[31] E. Ebrahimi, A. Sheykhi and H. Alavirad, Cent. Eur. J. Phys. DOI: 10.2478/s11534-013-0253-x.

[32] P.G. Bergmann, Int. J. Theor. Phys. 1 (1968) 25;

R.V. Wagoner, Phys. Rev. D 1 (1970) 3209;

K. Nordtvedt, Astrophys. J. 161 (1970) 1059.

[33] J.D. Bekenstein, Found. Phys. 16 (1986) 409.

[34] I.L. Shapiro, J. Sola, JHEP 0202 (2002) 006;

A. Babi c, B. Guberina, R. Horvat, H. tefanci c, Phys. Rev. D 65 (2002) 085002;

I.L. Shapiro, J. Sola, C. Espana-Bonet, P. Ruiz-Lapuente, Phys. Lett. B 574 (2003) 149.

[35] M. Reuter, Phys. Rev. D 57 (1998) 971;

A. Bonnano, M. Reuter, Phys. Rev. D 65 (2002) 043508.

[36] R. Horvat, Phys. Rev. D 70 (2004) 087301.

[37] B. Guberina, R. Horvat, H. Nikolic, Phys. Rev. D 72 (2005) 125011.

[38] G. S. Bisnovatyi-Kogan, Int. J. Mod. Phys. D 15 (2006) 1047.

[39] Damour T.,et al, Phys. Rev. Lett. 61 (1988) 1151.

[40] D.B. Guenther, Phys. Lett. B 498 (1998) 871.

[41] E. Gaztanaga, E. Garcia-Berro, J. Isern, E. Bravo and I. Dominguez, Phys. Rev. D 65 (2002) 023506.

[42] M. Biesiada, B. Malec, Mon. Not. R. Astron. Soc. 350 (2004) 644.

[43] S. Ray, U. Mukhopadhyay, Int. J. Mod. Phys. D 16 (2007) 1791.

[44] M. Jamil, E. N. Saridakis, M. R. Setare, Phys. Lett. B 679, 172, (2009).

[45] J. Lu, E. N. Saridakis, M. R. Setare, L. Xu, J. Cosmol. Astropart. Phys. 1003 (2010) 031.

[46] A. Sheykhi, M. R. Setare, Int. J Theor. Phys. 49 (2010), 2777.

[47] A. Sheykhi, Phys. Scrip. 85 (2012) 045901.

[48] R.A. Daly et al., Astrophysics J. 677 (2008) 1.

[49] E. Komatsu et al. [WMAP Collaboration], Astrophys. J. Suppl. 192, 18 (2011).

[50] V. Salvatelli, A. Marchini, L. L. Honorez and O. Mena, Phys. Rev. D 88 (2013) 023531. 
[51] T. Padmanabhan and S. M. Chitre, Phys. Lett. A 120 (1987) 433.

[52] I. Brevik and O. Gorbunova, Gen. Relativ. Gravit. 37 (2005) 2039.

[53] I. Brevik, O. Gorbunova and Y. A. Shaido, Int. J. Mod. Phys. D 14, 1899 (2005);

I. Brevik and O. Gorbunova, Eur. Phys. J. C 56 (2008) 425;

I. Brevik, Eur. Phys. J. C 56 (2008) 579.

[54] M. Cataldo, N. Cruz and S. Lepe, Phys. Lett. B 619, 5 (2005);

M. Szydlowski and O. Hrycyna, Annals Phys. 322 (2007) 2745.

[55] W. Zimdahl and D. Pavon, Phys. Rev. D 61 (2000) 108301.

[56] S. D'Innocenti, G. Fiorentini, G.G. Raffelt, B. Ricci, A. Weiss, Astron. Astrophys. 312 (1996) 345;

K. Umezu, K. Ichiki, M. Yahiro, Phys. Rev. D 72 (2005) 044010;

S. Nesseris, L. Perivolaropoulos, Phys. Rev. D 73 (2006) 103511. 